\title{
Estimation of Design Parameters of the Crank-Connecting Rod Mechanism of Engines for Mobile Agricultural Machines
}

\author{
$F$. Khaliullin*, G. Pikmullin, A. Nurmiev, $M$. Lushnov
}

Kazan State Agrarian University, Kazan, Russia

\begin{abstract}
An accurate choice of the design model of the crank-connecting rod mechanism of piston internal combustion engines affects the accuracy of the calculation results and their complexity. At present, most of scientists and technicians choose a two-mass design model to analyze the operation of the crankconnecting rod mechanism. The model considers only the rotational and reciprocating movements of two masses, which are connected by a rigid weightless rod. This model significantly simplifies the calculations, neglects the elastic deformations of the parts of the crank-connecting rod mechanism, and eliminates the need for compiling the equations of dynamics in partial derivatives. However, the model has a number of drawbacks. The calculation results obtained using the two-mass model exhibit significant errors, which mainly depend on the design features of the connecting rod assembly. The paper discusses multi-mass design models, where the connecting rod assembly can comprise several lumped masses located along its length. In this case, the plane-parallel motion of these masses is added. The masses have weightless and absolutely rigid bonds. Forces and moments acting on the piston assembly and the crank are calculated according to the equations compiled. Comparison of the calculation results with the results obtained for a two-mass model can be used to determine errors and choose a design model that provides the required accuracy. The considered design model is of interest to engineers and technicians engaged in the design and calculation of the crank-connecting rod mechanism of piston internal combustion engines.
\end{abstract}

\section{Introduction}

The production of agricultural products is characterized by the use of various mechanisms, which include devices for converting the rotary motion of the drive unit into the reciprocating motion of the working body. The correct choice of the design parameters of such devices, an adequate assessment of their effect on the effective operation of the entire mechanism is relevant; therefore, numerous scientific works are devoted to this issue.

In [1], the authors study the effect of the device damping properties on the dynamics of the mechanical part of the combine. In [2], the author substantiated the advantages and application areas of mobile robotic systems equipped with walking propellers. Prospects for further research are analyzed. The optimization involves estimation of the geometrical parameters of the walking mechanism, which ensure the movement of the supporting leg along a given trajectory [3-5].

In [6], the kinematic analysis of the slider-crank desaxial mechanism is presented for further synthesis of the mechanism with regard to the peculiarities of its use in the actuators of crank-connecting rod presses and mechanization units. In addition, the position function and transfer functions of the mechanism were determined. Dependences for solving the inverse problem of the mechanism analysis were provided. Precise and approximate dependencies were presented for calculating the kinematic characteristics of the mechanism. The effect of the degree of deaxiality on changes in the basic kinematic characteristics of the mechanism was considered.

The crank-connecting rod mechanism (CRM) is one of the most important mechanisms of modern technology, which is found in most internal combustion engines, including car and truck engines, as well as other small engines. In [7], the mathematical model of CRM is a nonlinear system of differential-algebraic equations (DAE) of the third order, and it is difficult to integrate it numerically. In the study, the differential transform method is proposed to obtain an approximate analytical solution of the CRM model in the form of converging series. Moreover, post-processing of the solution of the power series using the Padé resummation method is proposed to expand the region of convergence of the solution to the approximate series.

In [8-10], the slider-crank mechanism is a special four-bar linkage, which simultaneously provides both linear and rotary motion. The CAD model was prepared in CATIA V5 to simulate the mechanism and determine the exact path of the mechanism. The analytical method that can be used to determine various crank positions and the corresponding slider position in the slider-crank mechanism is discussed.

CRM occupies an especially important place in the design of piston internal combustion engines (ICE). The

\footnotetext{
Corresponding author: khaliullin_kai_adis@mail.ru
} 
ICE dynamics and, accordingly, the high-speed and load operation modes of the entire mechanism depend on the correct choice of the design parameters of the CRM.

To simplify the dynamic calculation of piston ICE, the actual CRM is replaced by a dynamically equivalent system of lumped masses. The problem in the dynamic calculation of the CRM is the determination of the inertial forces of masses performing complex motion (masses of the connecting rod). Therefore, in the dynamic study of the engine, an approximate method of determining the inertial forces of the connecting rod is adopted to replace, on the basis of the laws of mechanics, the motion of the actual mass of the connecting rod by the motion of two conventional masses, one of which conventionally slides, and the other one rotates [11].

Theoretically, the following conditions must be fulfilled to obtain a dynamically replacing system:

1) the sum of all replacement masses must be equal to the mass of the connecting rod;

2) the gravity center of all replacement masses must coincide with the gravity center of the connecting rod and move according to the law of motion of this gravity center;

3) the sum of the inertia moments of all replacement masses relative to the axis passing through the gravity center of the connecting rod should be equal to the inertia moment of the connecting rod relative to the same axis;

4) the angular acceleration of the replacing system in rotational motion with respect to its gravity center must be equal to the angular acceleration of the connecting rod in the same motion.

The third condition is typically not fulfilled. Due to the small numerical values of the difference between the moments of inertia of the reduced system and the connecting rod, which practically do not affect the accuracy of calculations, the third condition is neglected, and a system that satisfies the first two conditions is considered a dynamically replacing system [12].

At the same time, the vast majority of engines for modern cars and tractors are high-speed. The parts of the CRM develop significant speeds and accelerations, which in turn leads to increased requirements for the reliability of dynamic calculation, and to the emergence of alternatives in calculating the simplifications that were successfully used earlier $[13,14]$.

As an alternative, it is possible to calculate the forces acting in the CRM that bring the mass of the connecting rod to an equivalent four-mass model. Then it is necessary to compare the forces obtained by using the two- and four-mass models and to consider how the difference in forces will change depending on the change in the parameters of the given masses.

\section{Materials and Methods}

The tables presenting mass values of parts of the CRM or constructive masses can be used for an approximate determination of the mass of the piston $\mathrm{m}_{\mathrm{p}}$ and connecting rod $\mathrm{m}_{\mathrm{r}}$ in a two-mass model $[5,6]$.
Reduction to the two-mass model is carried out by dividing the distributed mass of the connecting rod into two lumped masses, one of which slides, and the other one rotates:

$$
m_{\mathrm{rp}}=m_{\mathrm{r}} \cdot \frac{L_{\mathrm{r}}-l^{I}}{L_{\mathrm{r}}} ; m_{\mathrm{rk}}=m_{\mathrm{r}} \cdot \frac{l^{I}}{L_{\mathrm{r}}}
$$

is the mass of the piston assembly:

$$
m_{p}=m_{p k}^{I} \cdot F_{p},
$$

where $F_{p}$ is the piston area;

are masses that reciprocate:

$$
m_{j}=m_{p} \cdot m_{r p}
$$

are masses that rotate:

$$
m_{R}=m_{k} \cdot m_{r k}
$$

\section{Results}

\section{Reduction to a four-mass model}

The connecting rod performs a complex plane-parallel motion. In three- and four-mass models, distributed inertial loads are considered much better, which increases the accuracy of numerical calculations [17, 18]. In addition, no additional moment from a pair of forces occurs with such a replacement, which arises in a two-mass model due to the fact that the moment of inertia of the reduced system is not equal to the moment of inertia of the connecting rod relative to its gravity center. During replacement of the connecting rod with a system of equivalent masses and further calculation, an approximate method of determining the inertial forces of the connecting rod is adopted. The mass of the connecting rod $m_{r}$ is conventionally reduced to three, four or more masses. One of these masses $m_{l}$ is located on the axis of the piston pin and refers to the translating parts, the other one $m_{2}$ is located on the axis of the connecting rod journal and refers to the rotating parts, $m_{3}$ and $m_{4}$ masses are located on the symmetry axis of the connecting rod.

The calculation will provide two types of substitutions with four-mass models (Figure 1): one for 'long' cranks and one for 'short' cranks. Long cranks are those installed in the CRM with $\lambda \leq 0.25$ or those in which the distance between the axes of the holes $L_{r}$ is more than three diameters of the connecting rod journals $D_{r j}$. Short cranks are those with $\mathrm{L}_{\mathrm{r}} \leq 3 \cdot \mathrm{D}_{\mathrm{rj}}$.

This is due to the fact that model 2 can be compared with a two-mass model, since the system can be reduced to the moment of inertia similar to that in the two-mass model [19]. For long cranks, model 1 is more suitable.

To better estimate the error in forces between different systems of mass substitution, we change the application points.

For model 1: the application point of $m_{u}$ varies within the interval $\mathrm{u}^{\mathrm{I}} \mathrm{u}^{\mathrm{II}}$, that is $l_{\mathrm{u}}=(0,2 \div 0,6) \cdot l^{I}$, the point application of $\mathrm{m}_{3}$ within the interval $\mathrm{k}^{\mathrm{I}} \mathrm{k}^{\mathrm{II}}$, that is $l_{3}=$ $(0,25 \div 0,66) \cdot l_{2}$. 

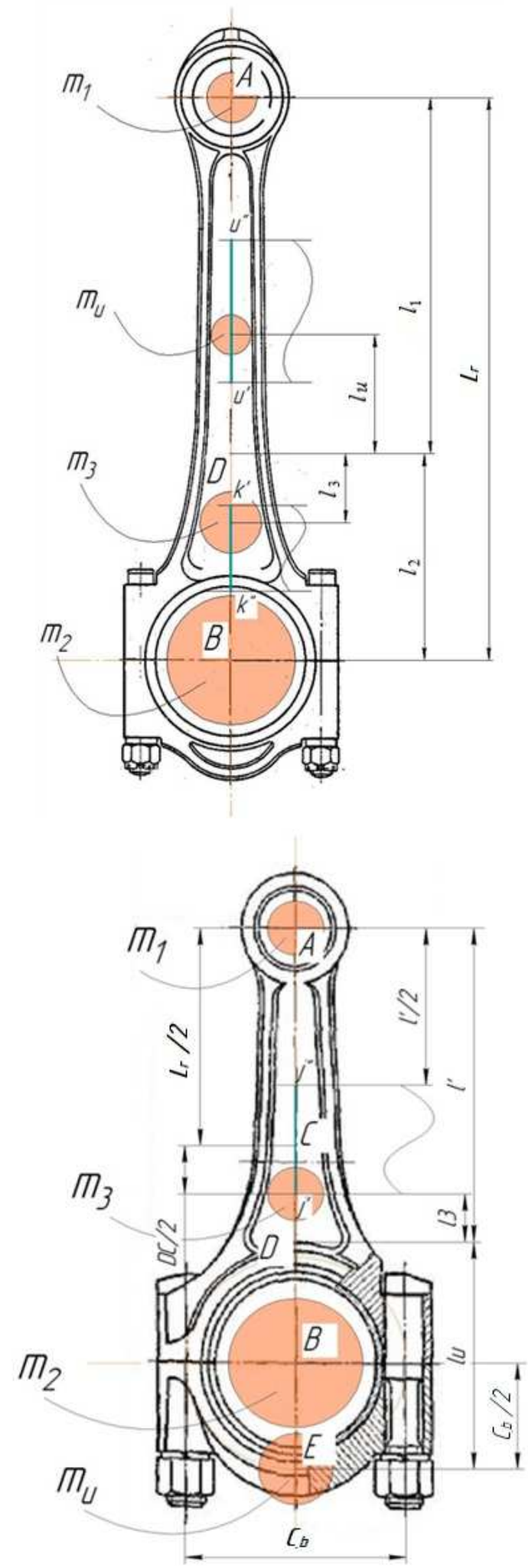

Figure 1. Distribution of masses in four-mass models, where $l^{I}$ is the distance from the piston head to the mass center; $l_{2}$ is the distance from the mass center to the axis of the connecting rod journal; $l_{u}, l_{3}$ are distances from the points of application of the masses $m_{u}$ and $m_{3}$, respectively, to the mass center; $D$ is the center of mass; $C$ is the middle of $L_{r} ; C_{b}$ is the distance between the axes of the cover fixing bolts; $I_{r}$ is the moment of inertia of the connecting rod. In this case, the length of the connecting rod (the length between the centers of the holes in the connecting rod) $L_{r}=l^{I}+l_{2}$. The masses $m_{3}$ and $m_{4}$ are typically smaller than $m_{1}$ and $m_{2}$.
For model 2: the application point of $m_{3}$ varies within the interval $\mathrm{j}^{\mathrm{I}} \mathrm{I}$, that is $l_{3}$ varies from the middle of the distance between the point of the mass center and the middle of $L_{r}$ to the middle of $l^{I}, l_{u}$ and remains constant and equal to:

$$
l_{u}=L_{r}-l^{I}+C_{b} / 2
$$

The condition for replacing the connecting rod with lumped masses is determined by the equality of masses, static moments and moments of inertia relative to the center of mass:

For model 1:

$$
\begin{aligned}
& m_{r}=m_{1}+m_{2}+m_{\mathrm{u}}+m_{3} \\
& m_{1} \cdot l^{I}+m_{\mathrm{u}} \cdot l_{\mathrm{u}}=m_{2} \cdot l_{2}+m_{3} \cdot l_{3} \\
& I_{\mathrm{r}}=m_{1} \cdot\left(l^{I}\right)^{2}+m_{2} \cdot l_{2}^{2}+m_{\mathrm{u}} \cdot l_{\mathrm{u}}^{2}+m_{3} \cdot l_{3}^{2}
\end{aligned}
$$

For model 2:

$$
\begin{aligned}
& m_{\mathrm{r}}=m_{1}+m_{2}+m_{\mathrm{u}}+m_{3} \\
& m_{1} \cdot l^{I}+m_{3} \cdot l_{3}=m_{2} \cdot l_{2}+m_{1} \cdot l_{1} \\
& I_{\mathrm{r}}=m_{1} \cdot\left(l^{I}\right)^{2}+m_{2} \cdot l_{2}^{2}+m_{\mathrm{u}} \cdot l_{\mathrm{u}}^{2}+m_{3} \cdot l_{3}^{2}
\end{aligned}
$$

In both cases, there are three equations and four unknowns. This means that one of the masses is to be set independently, and this mass in both cases is $m_{u}$. In the first case, it will be approximately equal to the mass of the rod section from the piston head to the center of mass (double-T-iron with a width $b=\min$ is the value of the rod width of the real connecting rod). In the second case, this mass is equal to $2 / 3$ of the mass of the connecting rod cover with bolts. These masses, as well as the points of their application, will vary during calculations $m_{u}, m_{3}$ $\approx(0,1 \div 0,18) \cdot m r$. Introduce a parameter that characterizes the ratio of the given mass $m_{u}$ to the mass of the connecting rod $m_{r}$ :

$$
z=\frac{m_{u}}{m_{r}} \approx(0.05 \div 0.20) \cdot m_{r}
$$

Transform the system of equations.

First, calculate the moment of inertia of the two-mass model:

$$
I_{r}=m_{r}\left(L_{r}-l^{I}\right) l^{I}
$$

Later, we will consider it. As mentioned above, when replacing the four-mass model of model 1 type, it is impossible to achieve the same moment of inertia, but let us not to take values that differ from it by more than $25 \%$. For the four-mass model of model 2 type, the moment of inertia remains the same. Let us introduce the ratio of the moments of inertia of the four-mass model to the two-mass one:

$$
q=\frac{I_{r 4 m}}{I_{r 2 m}} \approx(75 \ldots 95) \% \text { from } I_{r 2 m}
$$

For model 1:

-first transformation 


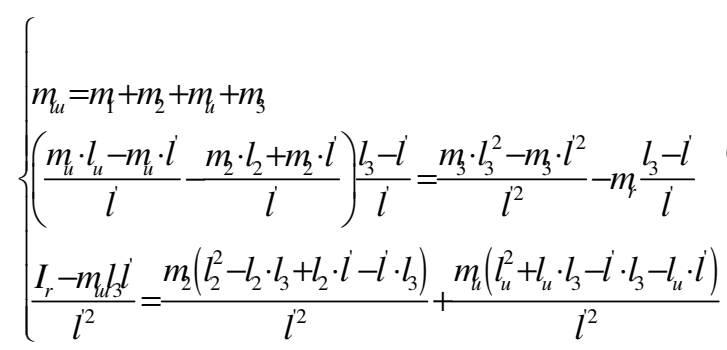

-second transformation:

$$
\begin{aligned}
& m_{2}=\frac{I_{r}-m_{r} l_{3} l^{\prime}-m_{u}\left(l_{u}^{2}+l_{u} \cdot l_{3}-l^{\prime} \cdot l_{3}-l_{u} \cdot l^{\prime}\right)}{\left(l_{2}^{2}-l_{2} \cdot l_{3}+l_{2} \cdot l^{\prime}-l^{\prime} \cdot l_{3}\right)} \\
& m_{3}=\frac{\left(m_{u} \cdot l_{u}-m_{u} \cdot l^{\prime}-m_{2} \cdot l_{2}-m_{2} \cdot l^{\prime}+m_{r}\right) \cdot\left(l_{3}-l^{\prime}\right)}{l_{3}^{2}-l^{2}}
\end{aligned}
$$

From the obtained system of equations:

$$
\begin{gathered}
m_{2}=\frac{I_{r}-m_{r} l_{3} l^{\prime}-m_{r}\left(l_{u}^{2}+l_{u} \cdot l_{3}-l^{\prime} \cdot l_{3}-l_{u} \cdot l^{\prime}\right)}{\left(l_{2}^{2}-l_{2} \cdot l_{3}+l_{2} \cdot l^{\prime}-l^{\prime} \cdot l_{3}\right)} \\
m_{3}=\frac{\left(m_{u} \cdot l_{u}-m_{u} \cdot l^{\prime}-m_{2} \cdot l_{2}-m_{2} \cdot l^{\prime}+m_{r}\right) \cdot\left(l_{3}-l^{\prime}\right)}{l_{3}^{2}-l^{2}} \\
m_{1}=m_{r}-m_{2}-m_{u}-m_{3} .
\end{gathered}
$$

The masses for model 2 are calculated similarly.

It should be noted that for the calculation according to model 1, the moment of inertia should be less than that of the connecting rod of the same mass and length in a two-mass model.

The inertial forces acting in the CRM (Fig. 5), in accordance with the motion of the reduced masses in a two-mass model, are divided into inertial forces of translating masses $P_{j}$ and centrifugal inertial forces of rotating masses $\mathrm{K}_{\mathrm{Rk}}$ and $\mathrm{K}_{\mathrm{R} r}$.

The force of inertia from reciprocating masses:

$$
\begin{gathered}
P_{j}=P_{j l}+P_{j I I}=-\left(m_{j} \cdot R \cdot \omega^{2} \cdot \cos \varphi+m_{j} \cdot R \cdot \omega^{2} \cdot \lambda \cdot \cos 2 \varphi\right) \\
P_{j I}=-m_{j} \cdot R \cdot \omega^{2} \cdot \cos \varphi,
\end{gathered}
$$

- first order force of inertia:

$$
P_{j I}=-m_{j} \cdot R \cdot \omega^{2} \cdot \lambda \cdot \cos 2 \varphi,
$$

- second order force of inertia.

These forces act along the cylinder axis and, similar to the gas pressure forces, are considered positive if directed towards the crank axis, and negative if directed away from the crank (Figure 2).

Centrifugal force of inertia of the rotating masses of the connecting rod:

$$
\mathrm{K}_{\mathrm{R}}=-\mathrm{m}_{\mathrm{rk}} \cdot \mathrm{R} \cdot \omega^{2} .
$$

The force is applied in the center of the connecting rod journal, is constant in magnitude and direction, and is directed along the crank radius.

\section{Force of inertia in a four-mass model}

We consider the plane-parallel motion of the connecting rod as the rotation of the connecting rod in a moving coordinate system with a reference point on the axis of the piston pin, and one of the axes of this system is directed along the cylinder axis. The translation of this moving coordinate system associated with the hole for the piston pin is conventionally relative to the stationary coordinate system associated with the top dead center of the piston $[19,20]$. Due to this motion of the connecting rod and reduction to the equivalent multi-mass model, the translational, centrifugal and tangential inertia forces of masses $m_{3}$ and $m_{u}$ (Figure 2) will be applied to the rod along with the inertia forces of translating masses $\mathrm{Pj}$ and the centrifugal inertia forces of rotating masses $K_{R K}$ and $\mathrm{K}_{\mathrm{R}} \mathrm{r}$ of masses $m_{3}$ and $m_{u}$. Let us consider the forces in order:

- Force of inertia of reciprocating masses. It acts along the cylinder axis and is subsequently added to the force of the gas pressure and yields the total force:

$$
P_{j}^{\prime}=-m_{j}^{\prime} \cdot R \cdot \omega^{2}(\cos \varphi+\lambda \cdot \cos 2 \varphi)
$$

where $m_{j}^{\prime}=m_{p k}+m_{1}$.

- Forces of inertia of masses $m_{3}$ and $m_{u}$ applied to the points of mass location and acting parallel to the cylinder axis:

$$
\begin{aligned}
& P_{j 3}=-m_{3} \cdot R \cdot \omega^{2} \cdot(\cos \varphi+\lambda \cdot \cos 2 \varphi), \\
& P_{j u}=-m_{u} \cdot R \cdot \omega^{2}(\cos \varphi+\lambda \cdot \cos 2 \varphi) .
\end{aligned}
$$

- Centrifugal forces of inertia of masses $m_{3}$ and $m_{u}$, applied to the points of location of these masses and acting along the axis of the connecting rod; these forces arise when the connecting rod rotates around the instantaneous center at the point of the piston head axis of the connecting rod:

$$
\begin{aligned}
& P_{u}^{n}=m_{u} \cdot a_{n}=m_{u}\left(l^{\prime} \mp l_{u}\right) \cdot \omega_{r}^{2}, \\
& P_{3}^{n}=m_{3} \cdot a_{n}=m_{3}\left(l^{\prime} \pm l_{3}\right) \cdot \omega_{r}^{2},
\end{aligned}
$$

in model 1 , the upper sign is put in the equations, that is, a minus for the 1 st equation and plus for the second one.

- Tangential forces of inertia of masses $m_{3}$ and $m_{u}$, applied to the points of location of these masses and acting perpendicular to the connecting rod axis:

$$
\begin{gathered}
P_{u}^{n}=-m_{u} \cdot a_{t}=m_{u} \cdot\left(l^{\prime} \mp l_{u}\right) \cdot \varepsilon_{r}, \\
P_{3}^{t}=-m_{3} \cdot a_{t}=m_{3} \cdot\left(l^{\prime} \pm l_{3}\right) \cdot \varepsilon_{r},
\end{gathered}
$$

signs are accepted according to the same principle as in the equations of centrifugal forces.

- Centrifugal force of inertia of rotating masses $m_{2}$ of the connecting rod. The force is applied in the center of the connecting rod journal, is constant in magnitude and direction and is directed along the crank radius:

$$
K_{R \emptyset}^{\prime}=-m_{2} \cdot R \cdot \omega^{2} \text {. }
$$




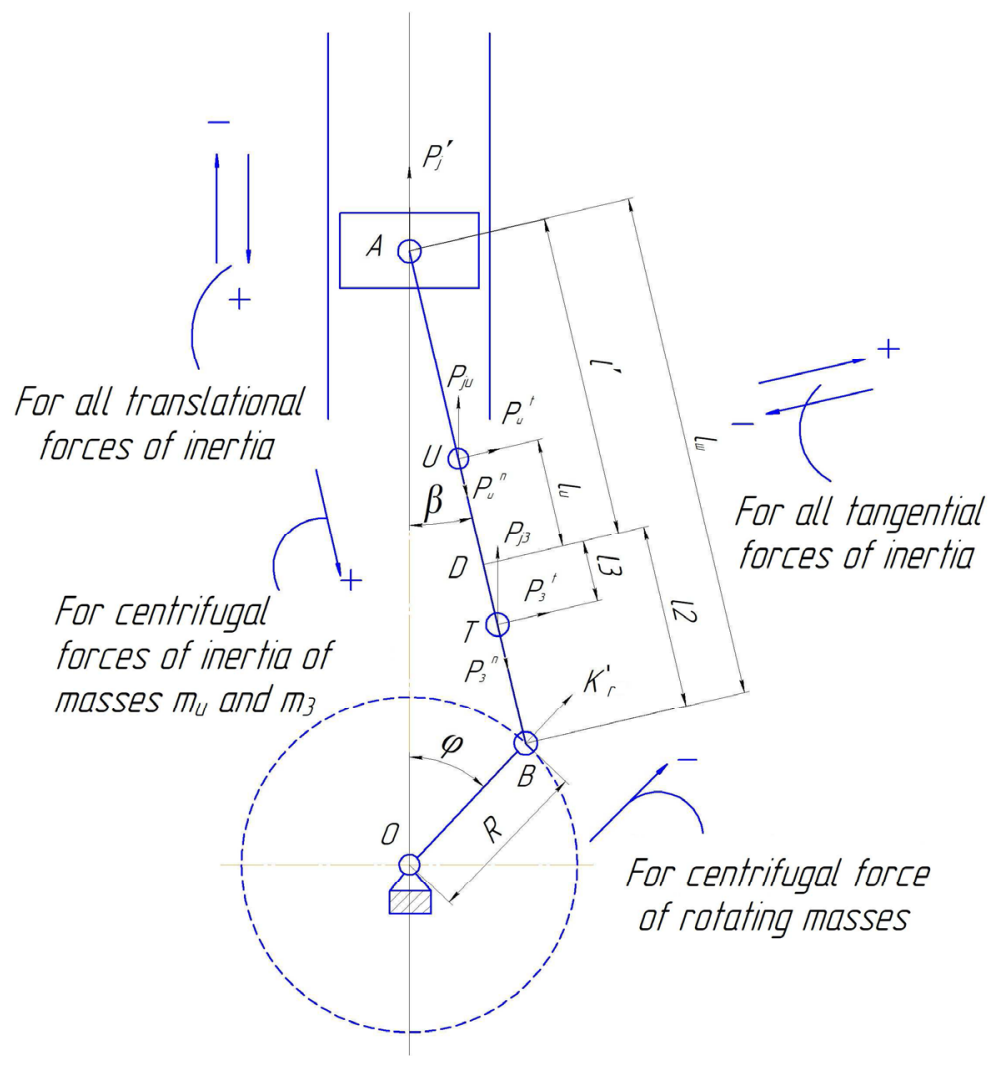

Total forces acting in the CRM.

Figure 2. Application of all inertial forces of all masses in a four-mass model.

To calculate the forces in four-mass models, consider the graphical arrangement of the forces applied to the connecting rod $[21,22]$. Let us choose the coordinate system with the origin at point $\mathrm{A}$ of the connecting rod and the $\mathrm{x}$-axis directed along the symmetry axis of the

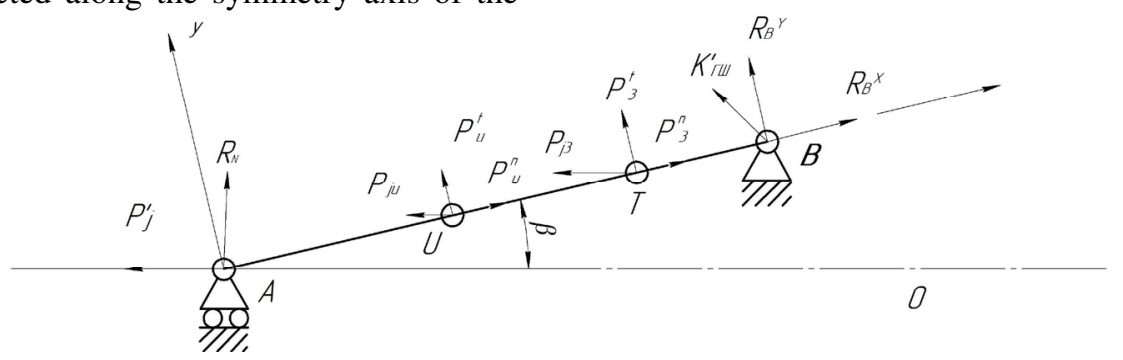

Figure 3. Scheme of the application of forces to the connecting rod in a four-mass replacement.

Let us find these support reaction forces. The magnitude and direction of the force $\mathrm{R}_{\mathrm{N}}$ is unknown, but it is known to act only perpendicular to the cylinder axis. Therefore, the scheme shows that the angle between the $y$-axis and this force is $\beta$. Translational forces of inertia act only parallel to the cylinder axis, which means that the angle between them and the $\mathrm{x}$-axis is $\beta$. The magnitude and direction of the force $R_{B}$ is unknown, therefore we conditionally replace it with two forces, one of which is directed perpendicular to the symmetry axis of the connecting rod, and the other one is parallel to it. Let us designate them $\mathrm{R}_{\mathrm{B}}{ }^{\mathrm{y}}$ and $\mathrm{R}_{\mathrm{B}}{ }^{\mathrm{x}}$, respectively.

Although the connecting rod is in motion, the forces of inertia applied to the rod enable considering it at a connecting rod. Replace the action of the side of the cylinder wall and the connecting rod journal on the piston by the reaction forces $R_{N}$ and $R_{B}$ (Figure 3). equations of statics can be applied:

$$
\begin{aligned}
& \sum M_{B}=-\left(P_{3}^{t}+P_{j 3} \cdot \sin \beta\right) \cdot\left(l_{2}-l_{3}\right)-\left(P_{u}^{t}+P_{j u} \cdot \sin \beta\right) . \\
& \left(l_{2}+l_{u}\right)-\left(R_{N} \cdot \cos \beta+P_{\Sigma}^{\prime} \cdot \sin \beta\right) \cdot L_{r}=0 .
\end{aligned}
$$

Calculate the force $\mathrm{R}_{\mathrm{N}}$ :

$$
R_{N}=\frac{-\left(P_{3}+P_{j 3} \cdot \sin \beta\right) \cdot\left(l_{2}-l_{3}\right)-\left(P_{u}^{i}+P_{j u} \cdot \sin \beta\right) \cdot\left(l_{2}+l_{u}\right)-P_{\Sigma}^{\prime} \cdot \sin \beta \cdot L_{r}}{\cos \beta \cdot L_{r}}
$$

The lateral force $\mathrm{N}^{\prime}$ acting from the piston side on the cylinder wall will be equal to $R_{N}$ with the opposite direction.

Calculate the force $\mathrm{R}_{\mathrm{B}}{ }^{\mathrm{y}}$ and $\mathrm{R}_{\mathrm{B}}{ }^{\mathrm{x}}$ :

$\sum M_{A}=\left(P_{3}+P_{j 3} \cdot \sin \beta\right) \cdot\left(i+l_{3}\right)+\left(P_{u}+P_{j u} \cdot \sin \beta\right) \cdot\left(i-l_{u}\right)+R_{B}^{v} \cdot L_{r}=0$ 


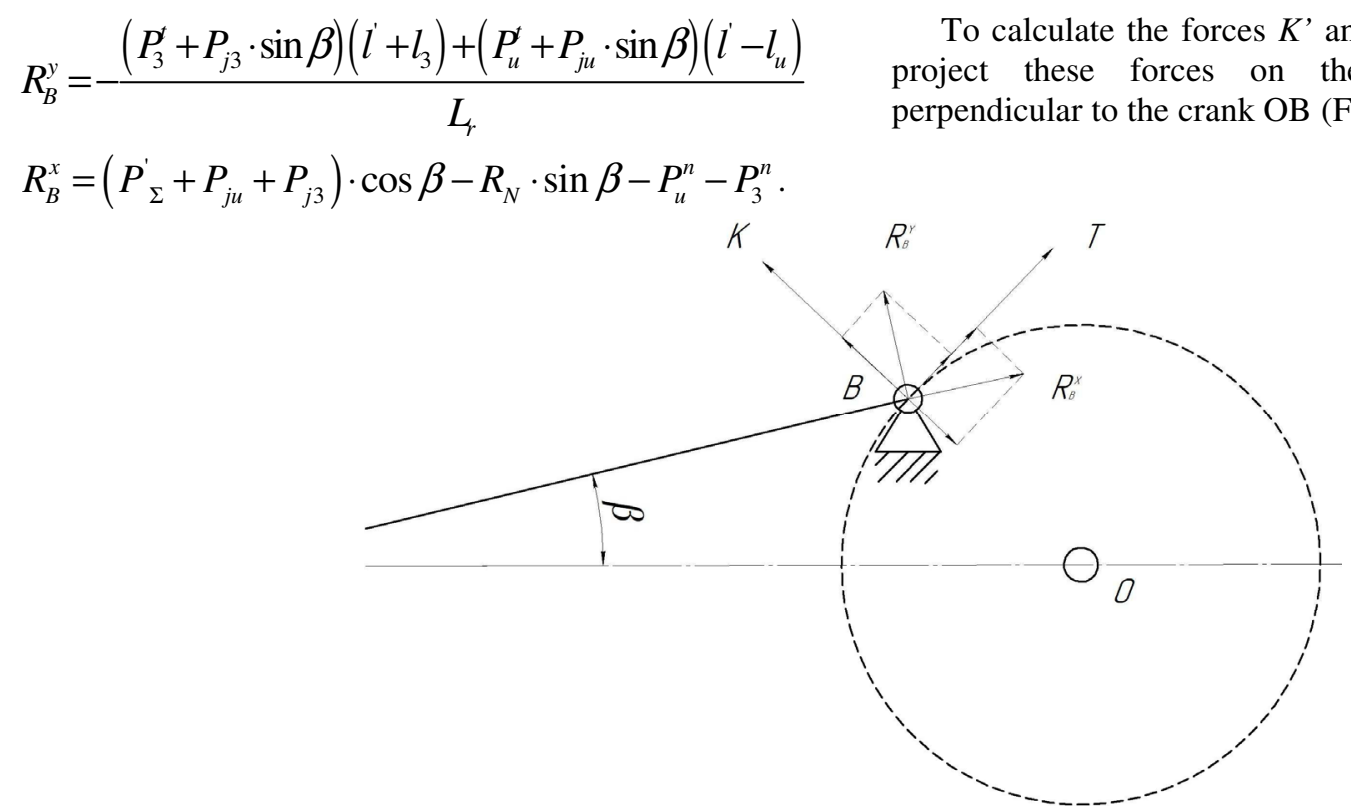

Figure 4. Projection of forces of the coordinate system associated with the connecting rod to the system associated with the crank.

$$
\begin{gathered}
K^{\prime}=-R_{B}^{y} \cdot(-\sin (\varphi+\beta))-R_{B}^{x} \cdot \sin (\varphi+\beta), \\
T^{\prime}=-R_{B}^{y} \cdot(\cos (\varphi+\beta))-R_{B}^{x} \cdot \sin (\varphi+\beta) .
\end{gathered}
$$

With regard to the force of inertia of rotating masses of the connecting rod, calculate the resulting force acting along the crank radius:

$$
K_{\text {sum }}^{\prime}=K^{\prime}+K_{R r}^{\prime}
$$

\section{Conclusion}

Let us take the above calculations to calculate and compare the forces in the two-mass and four-mass models of both types for several 'non-standard' connecting rods $[23,24]$. We use 'non-standard' connecting rods because an ordinary two-mass replacement model has quite acceptable errors for 'standard' rods. In our case, the connecting rods are nonstandard due to the displaced moment of inertia (either closer to the piston head, or closer to the lower connecting rod head) and length (too large or too small). In a four-mass model, the values of masses, the points of their application, and the moment of inertia will change. This is done to obtain the regularity of changes in the difference in forces and to assess the effect of the parameters. To better understand the calculation and its results, we introduce additional parameters that characterize the ratio of dynamically changing values in percent [25].

For comparison, we introduce the differences of the compared forces:

$$
\Delta N=\left|N_{2 m}-N_{4 m}\right|,
$$

$\Delta \mathrm{N}_{\max }$ is the maximum value of the difference.

$$
d N=\frac{\left|N_{2 m}-N_{4 m}\right|}{\left|N_{2 m}\right|} \cdot 100 \% .
$$

$\Delta \mathrm{T}, \Delta \mathrm{K}, \mathrm{dT}, \mathrm{dK}$ and the maximum values of these quantities are calculated similarly.

Let us set the initial data and sequentially consider the calculation results according to the initially specified order of this calculation. These data will be used to verify the calculation results.

Nominal speed:

Crank length:

Cylinder diameter: $\mathrm{n}=3000 \mathrm{rpm} / \mathrm{min}$

Piston stroke: $\mathrm{L}_{\mathrm{r}}=0.375 \mathrm{~m}$ $\mathrm{D}=0.15 \mathrm{~m}$

Nondimensional coefficient of CRM:

Crank mass: $\quad \mathrm{m}_{\mathrm{r}}=9.052 \mathrm{~kg}$

Mass of the reciprocating part of the crank: $m_{\mathrm{rp}}=3.800 \mathrm{~kg}$

Mass of the rotating part of the crank: $m_{\mathrm{rk}}=5.252 \mathrm{~kg}$

The ratio of the moment of inertia in a four-mass model to that in a two-mass model: $\mathrm{q}=90 \%$

The ratio of the specified mass $m_{u}$ to the mass of the connecting rod: $\mathrm{z}_{\mathrm{u}}=15 \%$

The indicator diagram values for the diesel engine.

A four-mass model of model 1 type.

-Calculation of the center of mass (distance from the axis of the piston head to the center):

$$
l^{\prime}=0,375 \cdot\left(1-\frac{3,8}{9,052}\right)=0,218 m \text {. }
$$

-Calculation of the moment of inertia for a two-mass model:

$$
I_{r}=9,052(0,375-0,218) 0,218=0,31 \mathrm{~kg} \cdot \mathrm{m}^{2} .
$$

-Calculation of masses with regard to the conditions for the equivalent reduction of masses:

$$
\begin{gathered}
m_{u}=1,36 \mathrm{~kg}, \\
m_{3}=0,043 \mathrm{~kg}, \\
m_{2}=4,7 \mathrm{~kg}, \\
m_{1}=2,95 \mathrm{~kg} .
\end{gathered}
$$

-Choice of the moment of inertia for a four-mass model: 


$$
I_{r 4 M}=0,263 \mathrm{~kg} \cdot \mathrm{m}^{2} .
$$

-Optimal choice of lengths:

$$
\begin{gathered}
l_{u}=0,0725 \mathrm{~m}, \\
l^{\prime}=0,218 \mathrm{~m}, \\
l_{2}=0,157 \mathrm{~m}, \\
l_{3}=0,0525 \mathrm{~m} .
\end{gathered}
$$

Figure 5 presents the graphs of changes in the values of the main forces calculated from the crank rotation angle.

The maximum relative deviations of force factors are presented in Table 1 .
Table 1. Differences in forces acting in CRM in a fourmass model.

\begin{tabular}{|l|c|c|}
\hline No. & Force factor & $\begin{array}{c}\text { Relative deviations for } \\
\text { a four-mass model, } \%\end{array}$ \\
\hline 1 & $T$ & 3,76 \\
\hline 2 & $K$ & $-7,16$ \\
\hline 3 & $N$ & 15,45 \\
\hline
\end{tabular}

The values of force factor deviations presented in the table indicate the need for a reasonable choice of the design model of the crank-connecting rod mechanism of piston engines.
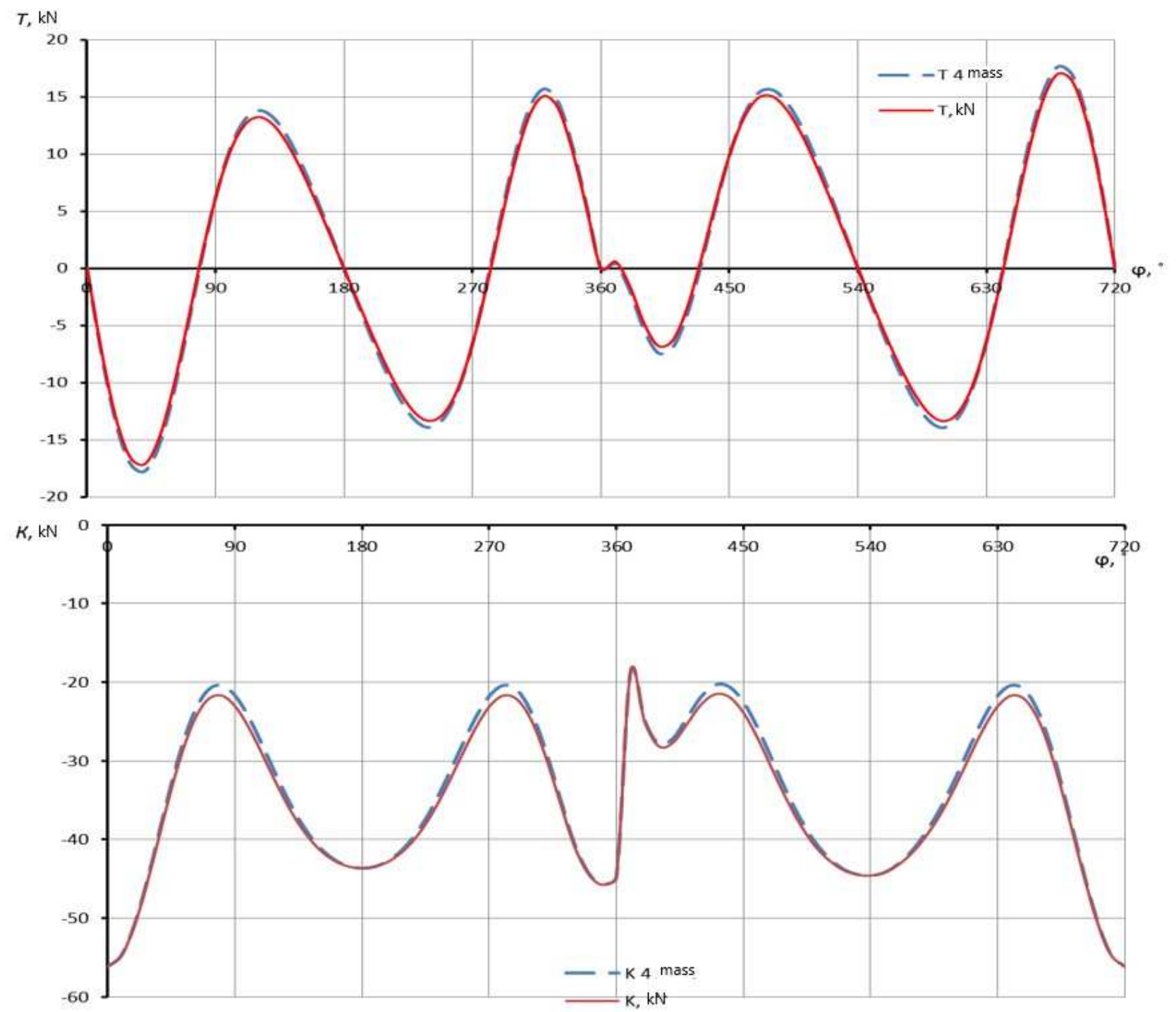


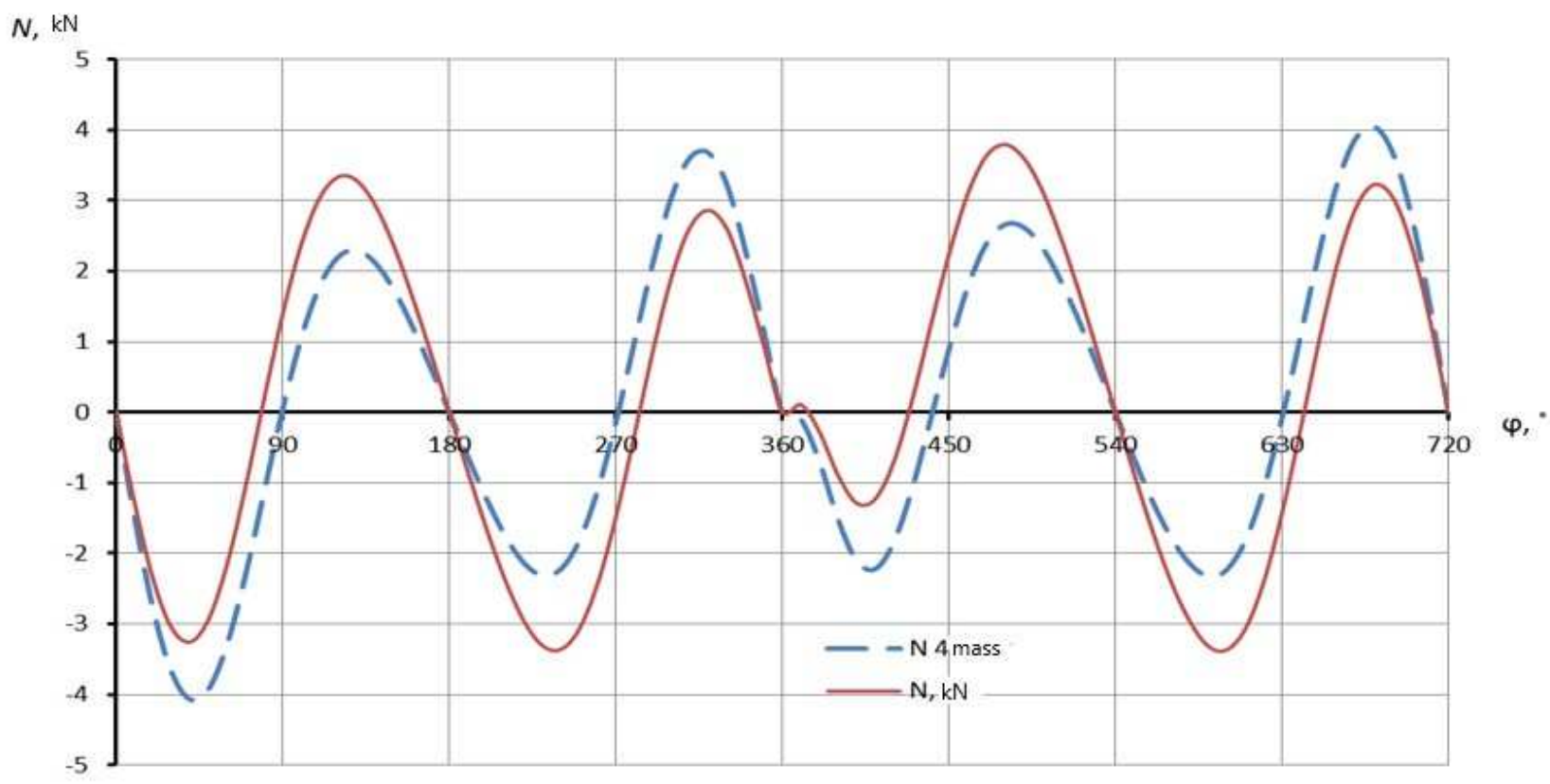

Fig. 5. Changes in forces $T, K, N$ for two- and four-mass design models.

\section{References}

1. A.S. Soloviev, Ya.P. Grinberg, V.S. Soloviev, Effect of damping properties of the engine on the dynamics of the mechanical part of the combine, Notes of Mining Institute, 178, 146 (2008)

2. V. Korendiy, Analysis of the structure and kinematics of a four-rod crank-swinging walking mechanism, Ukrainian journal of mechanical engineering and materials science, 1(2), 21-34 (2015)

3. A. Gassai, F. Choi, D. Whitaker, Design and optimization of crank mechanism (Pomona USA, 2011) $168 \mathrm{p}$.

4. N. Sansisi, R. Caminati, V. Parenti-Castelli, Optimal four-rod connection for stability and movement of human knee prostheses, XIX AIMETA Congress (Ancona, Italy, 2009) pp. 1-10

5. F. Klingener, Comparison of simple crank/slider mechanisms and rocker mechanisms (2011). Retrieved

from : http://demonstrations. wolfram.com/ComparingSimp leCrankSliderAndScotchYokeMechanisms

6. A. V. Yavtushenko, A. V. Yavtushenko, O. A. Kalantaeva, Kinematic characteristics of crank-crank presses with desaxial-rod crank mechanism, Modern equipment and technologies, 6 (2014). Retrieved from: https: //technology.snauka.ru/ru/ 2014/06/3806.

7. B. Benhammuda, H. Vasquez-Leal, Analytical solution of a nonlinear index-three DAE system that simulates a crank mechanism, Discrete dynamics in nature and society, 206473 (2015). DOI: https://doi.org/10.1155/2015/206473.
8. Alagala Hari Krishna, Design and kinematic analysis of the DMU crank mechanism of the slider, International Journal of Innovative Research in Advanced Development (IJIRAE) 1(9) (2014)

9. S. Erkaya, Ş. Su, I. Uzmai, Dynamic analysis of the crank-rod connecting mechanism with an eccentric connector and planetary gear, Theory of mechanisms and machines, 42(4), 393-408 (2007)

10. J.-L. Ha, R.-F. Fung, K.-Yu. Chen, S.-K. Xian, Dynamic Modeling and Crank Mechanism Identification, Journal of sound and vibration, 289(4-5), 1019-1044 (2006)

11. V.N. Lukanin, I.V. Alekseev, M.G. Shatrov et al., Internal combustion engines. Book 2. Dynamics and design (Higher school, Moscow, 1995) 320 p.

12. D.N. Vyrubov, S.I. Efimov, I.A. Ivaschenko, M.G. Kruglov et al., Internal combustion engines. Design and strength analysis of piston and combined engines (Mechanical engineering, 1984) $384 \mathrm{p}$.

13. I.V. Dovzhenko, Yu. I. Sennikov, L.V. Tuzov, Dynamic calculation of the crank-connecting rod mechanism of diesel engines, Propulsion engineering, 9, 18-21 (1983).

14. I.V. Dovzhenko, L.V. Kurmaz, Choice of the calculation model of the crank-connecting rod mechanism, Dep. in VIMI DN 1854 Sb. Riport, 17, 26 p. (1975)

15. S.P. Kosyrev, Dynamic loading of the crankconnecting rod mechanism of diesel engines, Engine manufacturing, 4, 23-26 (1980)

16. F. Khaliullin, A. Matyashin, M. Zemdikhanov, A. Martyushev, N. Davydov, Motorless pilot studies of crankshaft dampers of combustion engines, IOP

*Corresponding author: khaliullin_kai_adis@mail.ru 
Conference Series: Earth and Environmental Science, 699(1) 012041 (2021)

17. F. Khaliullin, G. Pikmullin, J. Aladashvili, D. Vakhrameev, E. Potapov, Identification of the optimal parameters of the torsional vibration damper of the internal combustion engine crankshaft for normal power settings, IOP Conference Series: Earth and Environmental Science, 699(1), 012042 (2021)

18. R.R. Shakirov, A.P. Inshakov, D.A. Vakhrameev, Improving the technical and economic indicators of the internal combustion engine operating in unsteady modes, Tractors and agricultural machines, 4, 28-31 (2011)

19. F. Khaliullin, R. Akhmetzyanov, F. Arslanov, Y. Korepanov, Method for determining the remaining life of engine by dynamic characteristics, Engineering for rural development, 19, 1096-1101 (2020)

20. F. Khaliullin, R. Shaikhutdinov, R. Shakirov, A. Ivanov, Taking into account construction parameters of crankshaft when evaluating characteristics of its equivalent torsion scheme, 19th International Scientific Conference Engineering for Rural Development (Jelgava, Latvia, 20-22 May 2020) pp. 1108-1114 (2020)

21. N. Egorov, F. Khaliullin, Z. Khaliullina, L. Zimina,
Adaptive support for power units of machine-tractor unit, 19th International Scientific Conference Engineering for Rural Development (Jelgava, Latvia, 20-22 May 2020) pp. 1737-1742 (2020)

22. I.G. Galiev, K.A. Khafizov, R.K. Khusainov, M.Kh. Faskhutdinov, Ensuring possibility of functioning of tractors in agricultural production taking into account residual resources of their units and systems, 19th International Scientific Conference Engineering for Rural Development (Jelgava, Latvia, 20-22 May 2020) vol. 18, pp. 4853 (2020)

23. F.Kh. Khaliullin, J.K. Aladashvili, A.A. Nurmiev, G.V. Pikmullin, S.A. Sinitsky, Determination of statistical data of conditional probabilities of the technical condition of internal combustion engines when compiling the Bayes diagnostic table, IOP Conference Series: Materials Science and Engineering, 635(1), 012017 (2019)

24. Liu Peng-Hong, Wang Ming $\mathrm{Wu}$, Mathematical model of the stress state of the crankshaft, Journal of Shanghai Jiaotong Univ., 7, 877-879 (1999)

25. A. R. Heath, P.M. McNamara, Crank stress analysis combination of finite element and classical analysis techniques, Trans. ASME. J. Eng. Gas Turbines and Power, 3, 268-275 (1990) 